\title{
Synthesis of 3D large pore germanosilicate zeolites using imidazolium-based long dications
}

\author{
Peng Lu, ${ }^{\text {abct }}$ Alvaro Mayoral, ${ }^{d}$ Luis Gómez-Hortigüela, ${ }^{e}$ Yaping Zhang, ${ }^{d}$ and Miguel A. \\ Camblor ${ }^{\text {a* }}$ \\ Instituto de Ciencia de Materiales de Madrid, Consejo Superior de Investigaciones Científicas \\ (ICMM-CSIC) c/ Sor Juana Inés de la Cruz 3, 28049 Madrid, Spain \\ bDalian National Laboratory for Clean Energy, Dalian Institute of Chemical Physics, Chinese Acad- \\ emy of Sciences, Dalian 116023, P.R. China \\ 'University of Chinese Academy of Sciences, Beijing 100049, P.R. China \\ ${ }^{d}$ Centre for High-resolution Electron Microscopy (CћEM), School of Physical Science and Technology, \\ ShanghaiTech University, 393 Middle Huaxia Road, Pudong, Shanghai 201210, China \\ eInstituto de Catálisis y Petroleoquímica, Consejo Superior de Investigaciones Científicas (ICP-CSIC), \\ c/ Marie Curie 2, 28049 Madrid, Spain
}

KEYWORD. Imidazolium-based dications, fluoride medium, zeolite beta polymorphs, germanosilicate, intergrowth, structure-directing agents.

\begin{abstract}
Introduction of small molar fractions of $\mathrm{Ge}$ in a synthesis system otherwise yielding the monodimensional large-pore zeolite MTW, produced three 3D large pore materials as the Ge fraction increased: *BEA, HPM-8 and HPM-7. HPM-8 is a zeolite consisting of an intergrowth of polymorphs D and E of the Beta family with a large predominance $(>80 \%)$ of polymorph D, according to DIFFaX simulations and High Resolution STEM analysis. After calcination, HPM-8 is remarkably stable upon contact with ambient air. HPM-7 likely possesses the POS topology, which is related to polymorph C. The density of double 4-ring units in the crystallized materials increases from *BEA to HPM-8 to HPM-7, i.e., as the Ge fraction increases. Interestingly, molecular mechanics show that in both HPM-7 and HPM-8 the organic dications prefer to site with their imidazolium rings in close contact with the D4R units as a consequence of a stronger confinement in this position. Indeed, the structure-directing role of these long dications can be explained as a consequence of the appropriate distance match between adjacent D4R units in both zeolite frameworks (and hence the negative charges associated to the occluded fluoride) and the imidazolium rings of these organic dications (and hence the positive charges), which is dictated by the length of the alkyl spacers, thus driving the crystallization pathway towards these particular germanosilicate zeolites.
\end{abstract}

\section{INTRODUCTION}

The large industrial applicability of zeolites relies on their wide variability of properties, which may afford fine tuning for optimal performance in a particular application. This variability is, in turn, the result of the large structural and chemical diversity of this class of materials. In the search for new zeolite structures, scientists use "structuredirecting" factors that may include the use of organic structure-directing agents (OSDA), ${ }^{1}$ use of fluoride, ${ }^{2,3}$ use of heteroatoms (i.e., atoms other than $\mathrm{Si}$ or Al that can enter tetrahedral sites of the framework) with a particular tendency to pro- duce structures with specific characteristics (double four membered ring units (D4R), spiro-5 units...). ${ }^{4}$ use of concentrated conditions, ${ }^{2}, 5-7$ etc. Frequently, an OSDA may fail in providing a strong structure-directing action, in which case a so-called "default structure" may crystallize, with the OSDA just serving as a space filler. ${ }^{4}$ A default structure is a zeolite that is pretty much stable and does not need a strong structure-direction to crystallize, and as a consequence they are obtained under a wide range of synthesis conditions. It goes without saying that default structures are, by definition, already known. When this 
is the case, one can combine several structure-directing factors to force the crystallization to devi-

This may be particularly the case when the default structure does not contain D4R. If under pure silica conditions a D4R-containing zeolite is produced, introduction of Ge will not likely produce a change in the phase selectivity, as recently shown for $\mathrm{AST}^{\underline{8}}$ and $\mathrm{STW}^{\mathrm{q}}$. However, when the default structure does not contain D4R an opportunity arises to crystallize different zeolites by departing away from the default structure by forcing the formation of D4R structures.

Here, we show that the crystallization of MTW, a 1D large pore zeolite that we consider a default structure in the synthesis of pure silica zeolites in the presence of fluoride anions because of its frequent occurrence (specially in diluted conditions typically favoring little specificity in structure-direction), ${ }^{4}$ can be totally avoided by using small fractions of $\mathrm{Ge}$, in which case different zeolites are obtained as the Ge fraction increases. The zeolites obtained in this way are all 3D large pore zeolites related to the zeolite Beta family: *BEA itself, HPM-8 and HPM-7. HPM-8 is an intergrowth of polymorphs $D$ and $E$ of the Beta family, similar to the recently reported SU-78 but with two noticeable differences: first, it is more abundant in polymorph D (over $80 \%$, compared to a $50: 50$ ratio in SU-78), and second, it is highly stable after calcination and exposure to ambient air. On the other hand, HPM-7 is likely isostructural to the recently reported PKU-16 (POS), which is related to polymorph C.

\section{EXPERIMENTAL SECTION}

\section{Synthesis of the OSDAs}

The imidazolium-based dications used in this work were synthesized by reacting 1,2dimethylimidazole with the corresponding linear $\alpha, \omega$-dibromoalkane with $\mathrm{n}=8$ and 10 carbon atoms, forming a doubly-charged cation with the imidazolium moieties on each end of the methylene chain, denoted as 8BDMI and 10BDMI, respectively. A typical synthesis route is as follows. $0.14 \mathrm{~mol}(13.733 \mathrm{~g})$ of 1,2-dimethylimidazole (Sigma-Aldrich, 98\%) was dissolved in $50 \mathrm{ml}$ chloroform (Sigma-Aldrich, 99\%) in a flask under magnetic stirring until completely dissolved, then $0.07 \mathrm{~mol}$ of the corresponding linear alkyl bromide (Sigma-Aldrich, $98 \%$ for $\mathrm{n}=8$, and $97 \%$ for $\mathrm{n}=10$ ) was added followed by another $50 \mathrm{ml}$ of chloroform (Sigma-Aldrich, 99\%). The above mixture was refluxed for 4 days and the solvent was removed by rotary evaporation to obtain the corresponding bromides (white solid for $\mathrm{n}=8$, thick transparent oil for $\mathrm{n}=10$ ).

${ }^{1} \mathrm{H}$ and ${ }^{13} \mathrm{C}$ NMR spectra of the bromides dissolved in $\mathrm{D}_{2} \mathrm{O}$ confirmed that the products synthesized were 8BDMI and 10BDMI (Figure S1 and S2). The dibromide salts were converted into their corresponding hydroxides by anion exchange. Dowex anion exchange resin 550A $(\mathrm{OH})$ (SigmaAldrich, 1.1 mequiv./1 ml) was washed with ate from the default structure and produce another, hopefully new and interesting zeolite. ${ }^{4}$

deionized water until no ammonia smell, then, the dibromide salts were dissolved in the resinwater mixture, which was kept under mild stirring overnight. The hydroxide solution was recovered by washing and filtration with deionized water and finally concentrated using rotoevaporation at $50{ }^{\circ} \mathrm{C}$. The final concentration of the hydroxide solution was determined by titration using phenolphthalein as indicator.

\section{Synthesis of zeolites}

The starting gels for the synthesis of germanosilicates were prepared by adding $\mathrm{GeO}_{2}$ (SigmaAldrich, 99.998\%) in the aqueous solution of the organic dication in a $50 \mathrm{ml}$ plastic beaker, then tetraethylorthosilicate (TEOS, 98\%, SigmaAldrich) was added. The mixture was magnetically stirred under room temperature overnight and then heated in a water bath until all the ethanol produced from the hydrolysis of TEOS plus some water (monitored by weight) were evaporated. In some cases, extra water was added in order to achieve the desired $\mathrm{H}_{2} \mathrm{O} / \mathrm{TO}_{2}$ ratio, where $\mathrm{T}$ stands for both $\mathrm{Si}$ and $\mathrm{Ge}$. Hydrofluoric acid (HF, Sigma-Aldrich, 48\%) was added and the mixture was homogenized with a spatula by hand for $15 \mathrm{~min}$. The final gel composition was (1x) $\mathrm{SiO}_{2}: x \mathrm{GeO}_{2}: 0.25 \mathrm{R}(\mathrm{OH})_{2}: 0.5 \mathrm{HF}: 5.0 \mathrm{H}_{2} \mathrm{O}$, where $0 \leqq x \leqq 0.4$.

The resulting thick gel was transferred into a Teflon liner, which was sealed into a stainless steel autoclave. The hydrothermal reaction was carried out by heating the autoclaves while tumbling $(\sim 60 \mathrm{rpm})$. The reaction temperature used was $150{ }^{\circ} \mathrm{C}, 175{ }^{\circ} \mathrm{C}$ or $190{ }^{\circ} \mathrm{C}$. The autoclaves were taken out at different time intervals and the solid product was recovered, washed with deionized water, and dried at $100{ }^{\circ} \mathrm{C}$. The encapsulated organics could be removed by calcination at 600 ${ }^{\circ} \mathrm{C}$ for $5 \mathrm{~h}$ in air.

\section{Characterization techniques}

The solid products were identified using Powder $X$-ray diffraction (PXRD) data obtained in a Bruker D8 Advance diffractometer using $\mathrm{Cu} \mathrm{K} \alpha$ radiation $(\lambda=1.5418 \AA)$. The amount of organics occluded in the as-made zeolites was determined by CHN elemental analysis using a LECO CHNS-932 analyzer. Thermal gravimetric analysis was carried out in a SDT Q600 TA instrument under air flow (100 ml/min) heating from $25^{\circ} \mathrm{C}$ to $1000{ }^{\circ} \mathrm{C}$ (with a heating rate of $10^{\circ} \mathrm{C} / \mathrm{min}$ ). Routine field emission scanning electron micrographs (FE-SEM) were collected using Philips XL30 S-FEG. Multinuclear magic angle spinning (MAS) NMR spectroscopy of as-synthesized samples was obtained at room temperature on a Bruker AV-400-WB equipment and the details have been given elsewhere. $\frac{10}{.}$ Fourier Transform infrared spectra were obtained with a Bruker IFS 66/S spectrophotome- 


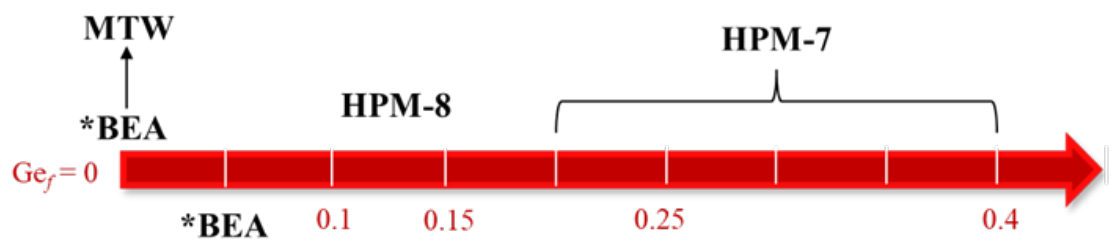

Figure 1. The as-made zeolite types obtained with 8BDMI as a function

of $\mathrm{Ge}_{f}$.

ter using the $\mathrm{KBr}$ pellet technique. The $\mathrm{N}_{2}$ adsorption/desorption isotherms were obtained at 77.35 K using a Micromeritics ASAP 2010 equipment.

\section{Computational details}

Molecular mechanics simulations were performed in order to find the most stable location of 8BDMI within HPM-7 (POS framework) and polymorph D of zeolite beta (as a representation of HPM-8). Simulations were carried out using the Forcite module implemented in Materials Studio software.11 The geometry of the zeolite structures were kept fixed during the calculations. Molecular structures and the interaction energies of the organic SDA cations with the frameworks were described with the CVFF forcefield. $\underline{12}$ The atomic charge-distribution of the dications was obtained from DFT+D calculations, using the B3LYP hybrid functional and the ESP charge calculation method, setting the total net charge to +2 . The positive charge of the organic dications was compensated by the framework by reducing the atomic charge of Si from 0.6 until neutrality. Framework oxygen charges were kept fixed to 0.3 . For studies with the POS framework (taken from the database of zeolite structures) ${ }^{13}$, depending on the case $1 \times 1 \times 1$ or $1 \times 1 \times 2$ supercells have been used as the zeolite model, while for polymorph D of zeolite beta, $\underline{14} 2 \times 2 \times 2$ supercells were used. The organic dications were manually docked in the framework in the required position/ orientation, and the most stable locations were obtained by simulated annealing. Relative energies are referred to the most stable case, and are given in $\mathrm{kcal} / \mathrm{mol}$.

\section{HRSTEM}

Transmission Electron Microscopy observations were performed in a double corrected JEOL GrandARM 300 which was operated at $300 \mathrm{kV}$. The microscope was equipped with two separated spherical aberration correctors for both TEM and STEM imaging conditions assuring in each case $0.7 \AA$ point resolution. It was also equipped with a JEOL EDS detector and a Gatan Quantum Energy Filter (GIF) for spectroscopic analyses. For every experiment presented here, the microscope was operated in Scanning Transmission Electron Microcopy (STEM) mode, collecting the electrons simultaneously using an Annular Dark Field (ADF) detector and an Annular Bright field (ABF) detector. Prior to observation, the materials were deeply crushed using mortar and pestle for up to 5 minutes with the intention of breaking the sample into very thin crystals which allowed obtaining high resolution images. The finely divided powder was dispersed in ethanol and sonicated and few drops of the suspension were deposited onto holey carbon copper microgrids. Before any electron microscopy (EM) experiments, the corrector was aligned using amorphous carbon assuring that the maximum spatial resolution could be achieved. Due to the high sensitivity of zeolites under the electron beam, STEM low dose conditions were necessary in order to acquire high resolution images with atomic-resolution. For this purpose, the exposure time did not exceed $8 \mathrm{sec}$ onds for the high-resolution data, with an image size of $1024 \times 1024$ pixels.

3D-electron diffraction tomography (3D-EDT) data was collected in a JEOL 2100 Plus (200 kV) transmission electron microscope using the software EDT Collect from AnaliteX company. ${ }^{15}$ The data was collected with a 0.2 degree increment and a total angle of 120 degrees.

High resolution scanning electron microscopy (SEM) was performed in a JEOL 7800 Prime. In order to achieve high-resolution images we use the GBSH (gentle beam super high) mode. In this mode, the gun voltage applied was $5.5 \mathrm{keV}$ with a decelerating voltage $-5 \mathrm{keV}$ on the sample holder, giving a final landing energy of $0.5 \mathrm{keV}$ on the samples.

\section{RESULTS AND DISCUSSION}

\section{Zeolite synthesis}

Table S1 summarizes the synthesis conditions and products obtained using 8BDMI and 10BDMI as SDAs under varying germanium molar fractions $\left(\mathrm{Ge}_{f}=\mathrm{Ge} /(\mathrm{Si}+\mathrm{Ge})\right)$. Additionally, the phase selectivity is schematically shown in Figure 1. The synthesis of zeolites under pure silica compositions was extensively reported recently for these dications of varying length: at high concentration 8BDMI produced zeolite *BEA that subsequently transformed into MTW, whereas 10BDMI yielded MWW that transformed also into MTW. $\underline{6}$ Lower concentrations directly yielded MTW in both cases. When Ge was introduced into the gel using 8BDMI as SDA three 3D large pore zeolites, *BEA, HPM-8 and HPM-7, were obtained with the increase of $\mathrm{Ge}_{f}$. The syntheses trials using 10BDMI with $\mathrm{Ge}_{f}$ at 0.1 and 0.25 also produced HPM-8 and HPM-7, respectively. 
As it is well known, *BEA is an intergrowth of two closely related polymorphs: polymorph $A$ and polymorph B. ${ }^{16}$ As for HPM-8, it is an intergrowth of polymorphs $D$ and $E$ of the same family, which are the remaining two hypothetical polymorphs of zeolite beta predicted by Burton et al. ${ }^{17}$ and successfully synthesized later by Yu et al. ${ }^{14}$ as SU-78, an intergrowth of both polymorphs in a close to 1:1 ratio. All the polymorphs of the Beta family contain a 3D system of interconnected large $12 \mathrm{MR}$ pores. HPM-8 appears to be much closer to polymorph $D$ than SU-78 (see below). We observed two types of XRD patterns for HPM-8 (Figure 2): The most frequent one presented somehow broader peaks and a merge of the two central most intense peaks. This difference initially suggested HPM-8 could present different degrees of disorder, however this does not appear to be the case (see below). In the case of HPM-7, a phase with a much similar XRD pattern was recently reported and named PKU-16 (POS), which is structurally related to polymorph $C$ of Beta by rotating half of the four-rings in double $m t w$ units resulting in a unique three dimensional $11 \times 11 \times 12$ channel system. ${ }^{18}$

The synthesis temperature played a vital role for the crystallization of HPM-7 as demonstrated in Figure S3. III-crystallized HPM-7 with amorphous phase was obtained at $150{ }^{\circ} \mathrm{C}$ after 2 days, while products with well-resolved patterns were obtained at $175^{\circ} \mathrm{C}$ and $190{ }^{\circ} \mathrm{C}$ under the same synthesis time. Prolonging the crystallization time rendered better-resolved patterns for all three synthesis batches. However, the reflections are still very broad for the products at $150{ }^{\circ} \mathrm{C}$, suggesting that the crystallization of HPM-7 may have a relatively high activation energy.

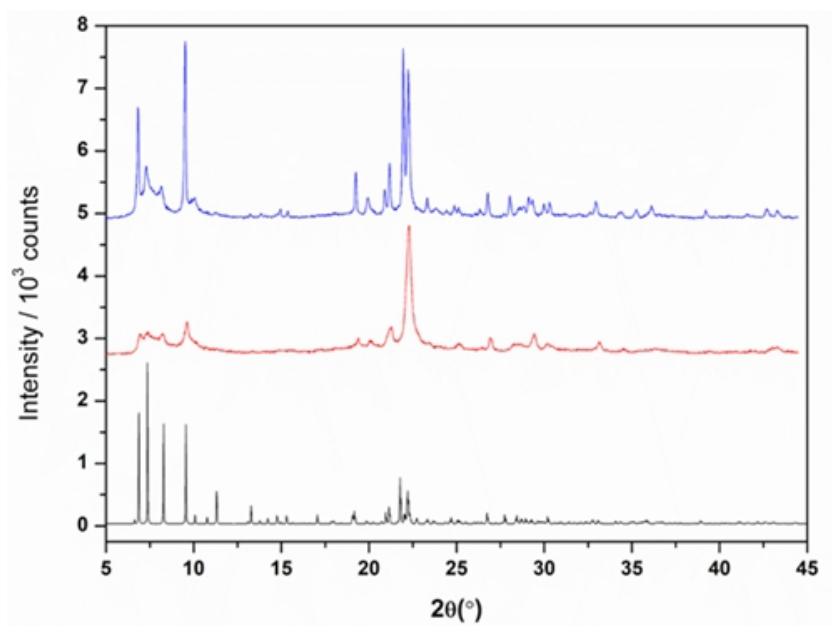

Figure 2. The XRD patterns of as-made HPM-8 synthesized with 8BDMI and $\mathrm{Ge}_{f}=0.15$ in comparison with the simulated pattern of SU-78B (bottom trace). Two HPM-8 samples with markedly differently resolved patterns are shown.
The structure-directing ability of $8 \mathrm{BDMI}$ and 10BDMI towards various beta-related structures at different $\mathrm{Ge}_{f}$ suggests a significant altering role of $\mathrm{Ge}$ on the T-O-T connections. It has been long recognized that Ge prefers to locate in the D4R or D3R units especially in fluoride medium. ${ }^{8,19-21}$ Indeed, in going from *BEA (no D4R units in polymorphs $A$ or $B$ ) to HPM-8 (one D4R in a unit cell of $32 \mathrm{~T}$ atoms) and then to HPM-7 (four D4R per cell of $64 \mathrm{~T}$ atoms) the density of D4R units increases. Similar phenomenon of phase change along germanium fraction variation have been observed previously in other germanosilicate zeolites synthesis. ${ }^{5,22}$ On the other hand, the selectivity of the long dications used in this work towards Beta-related zeolites contrasts with the tendency of shorter dications to produce STW, which must be clearly due to the size of 8BDMI and 10BDMI being too large to accommodate the imidazolium moieties into two consecutive stw cages. $\frac{5}{}$

\section{Zeolite characterization}

The XRD patterns of HPM-8 in comparison with the simulated pattern of polymorph $\mathrm{D}$ of the Beta family (i.e., SU-78B) are shown in Figure 2. The peak intensity increased after calcination for both HPM-8 samples, which is especially obvious at the low angle region, and also, some additional peaks appeared in the region between $12^{\circ}$ and $16^{\circ}$ (Figure 3 ). The as-made HPM- 8 was indexed in the monoclinic system using DICVOL $6,{ }^{23}$ with unit cell parameters $a^{\prime}=c=13.9090 \AA, b^{\prime}=b=12.6815$ $\AA, c^{\prime}=a=12.5224 \AA, \beta=107.768^{\circ}$ and cell volume of $2103.44 \AA^{3}$, which are close to those previously reported for SU-78. SU-78 was synthesized with two SDAs, namely $\mathrm{N}$-ethyl-N-methyl-dicyclohexylammonium hydroxide and $\mathrm{N}, \mathrm{N}$ dimethyl-dicyclohexylammonium hydroxide, which are mono-cationic organics, in contrast with our imidazolium-based dications. Interestingly, HPM-8 shows much higher stability at room temperature against moisture than SU-78, which collapsed after being exposed in air for $1 \mathrm{~h}$ after calcination. $\underline{14}$ The XRD patterns of calcined HPM- 8 after exposure to ambient air for different periods of time demonstrates its improved stability (Figure 3), which could give HPM-8 a high potential for catalytic applicability. The stability difference between our HPM-8 and SU-78 may be due to the lower Ge content in HPM-8 $\left(\mathrm{Ge}_{f}=0.1-0.15 \mathrm{com}\right.$ pared to 0.3). The chemical analysis of the HPM-8 material has been performed by means of X-ray Dispersive Spectroscopy using both Transmission and Scanning electron microscopes using at least 50 different crystallites. The obtained Si/Ge ratio was 7.3 , resulting in a Gef $=0.145$ for a sample synthesized from a gel with $\mathrm{Ge}_{\mathrm{f}}=0.1$, confirming the much lower $\mathrm{Ge}_{f}$ value of HPM-8 compared to SU-78. 
Our as-made HPM-7 exhibits a similar pattern to the simulated pattern of PKU-16 as shown in Figure 4. Different from PKU-16, synthesized using the relatively small neutral amine 4-methylaminopyridine (DMAP) as the SDA, HPM-7 was synthesized with long imidazolium-based dications. The corresponding calcined HPM-7 samples (Figure S4) show increased intensities at the low angle region, a normal phenomenon for the calcined zeolites indicating the release of the occluded organics. Additionally, new peaks showed up at about $13^{\circ}$ and $15^{\circ}$. Unfortunately, the stability of HPM-7 upon calcination and subsequent exposure to ambient air is significantly lower than that of HPM-8, which we attribute to its increased $\mathrm{Ge}_{f}$; while freshly calcined HPM-7 still presents a good crystallinity, exposure to ambient air significantly destroys the framework in a relatively short time (Figure S4).

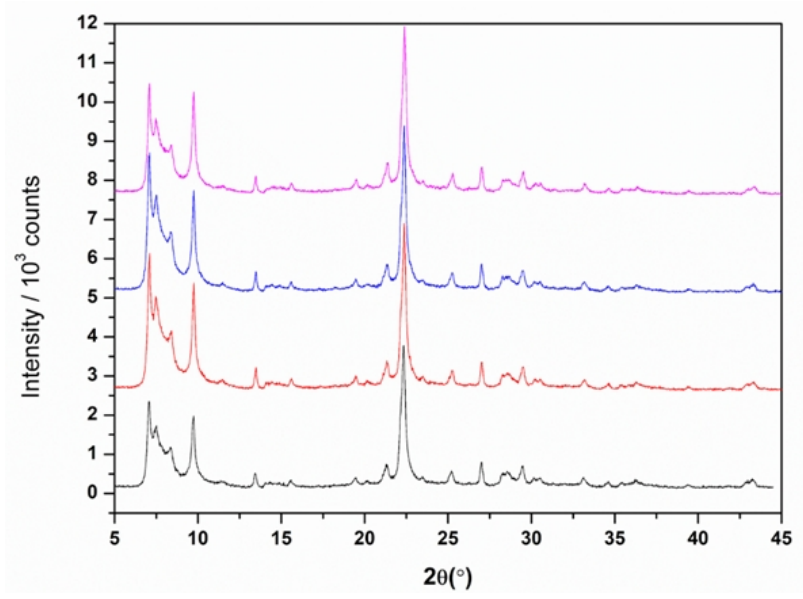

Figure 3. The XRD patterns of calcined HPM-8 (from bottom to top): freshly calcined, stored in closed eppendorf for 3 months, after $3 \mathrm{~h}$ in open air and after $3 \mathrm{~d}$ in open air. 
Table 1. Chemical analysis results of as-made zeolites.

\begin{tabular}{|c|c|c|c|c|c|c|c|c|c|c|}
\hline SDA & Phase & $\mathrm{C}(\%)$ & $H(\%)$ & $N(\%)$ & & $\mathrm{C} / \mathrm{N}^{\mathrm{a}}$ & & $\mathrm{H} / \mathrm{N}^{\mathrm{a}}$ & $\mathrm{TG}^{\mathrm{b}}$ & empirical composition ${ }^{c}$ \\
\hline 8BDMI & *BEA & 11.80 & 1.97 & 2.78 & ) & $4.95(4.5$ & & $9.85(8.0)$ & $80.47(82.8)$ & $\left|\mathrm{C}_{18} \mathrm{H}_{32} \mathrm{~N}_{4} \mathrm{~F}_{2}\right|_{2.30}\left[\mathrm{SiO}_{2}\right]_{64}[\mathrm{H} 2 \mathrm{O}]_{0.71}$ \\
\hline 8BDMI & *BEA & 12.57 & 1.95 & 2.78 & ) & $5.27(4.5$ & & $9.75(8.0)$ & $79.1(82.8)$ & $\begin{array}{c}\left|\mathrm{C}_{18} \mathrm{H}_{32} \mathrm{~N}_{4} \mathrm{~F}_{2}\right| \\
{ }_{2.38}\left[\mathrm{Si}_{0.95} \mathrm{Ge}_{0.05} \mathrm{O}_{2}\right]_{64}\left[\mathrm{H}_{2} \mathrm{O}\right]_{0.67}\end{array}$ \\
\hline 8BDMI & ${ }^{d} \mathrm{HPM}-8$ & 12.31 & 1.91 & 2.90 & ) & $4.95(4.5$ & & $9.15(8.0)$ & $80.0(82.1)$ & $\begin{array}{c}\left|\mathrm{C}_{18} \mathrm{H}_{32} \mathrm{~N}_{4} \mathrm{~F}_{2}\right| \\
{ }_{2.60}\left[\mathrm{Si}_{0.90} \mathrm{Ge}_{0.10} \mathrm{O}_{2}\right]_{64}\left[\mathrm{H}_{2} \mathrm{O}\right]_{0.45}\end{array}$ \\
\hline 8BDMI & HPM-8 & 11.36 & 1.81 & 2.85 & ) & $4.65(4.5$ & & $8.83(8.0)$ & $81.2(82.5)$ & $\begin{array}{c}\left|\mathrm{C}_{18} \mathrm{H}_{32} \mathrm{~N}_{4} \mathrm{~F}_{2}\right| \\
2.63\left[\mathrm{Si}_{0.85} \mathrm{Ge}_{0.15} \mathrm{O}_{2}\right]_{64}\left[\mathrm{H}_{2} \mathrm{O}\right]_{0.32}\end{array}$ \\
\hline 8BDMI & HPM-7 & 11.04 & 1.76 & 2.43 & ) & $5.30(4.5$ & ) & $10.06(8.0$ & $82.6(84.9)$ & $\begin{array}{c}\left|\mathrm{C}_{18} \mathrm{H}_{32} \mathrm{~N}_{4} \mathrm{~F}_{2}\right| \\
2.32\left[\mathrm{Si}_{0.75} \mathrm{Ge}_{0.25} \mathrm{O}_{2}\right]_{64}\left[\mathrm{H}_{2} \mathrm{O}\right]_{0.78}\end{array}$ \\
\hline 10BDMI & ${ }^{\mathrm{d}} \mathrm{HPM}-8$ & 12.43 & 1.99 & 2.76 & ) & $5.25(5.0$ & ) & $10.02(9.0$ & $80.6(81.6)$ & $\begin{array}{c}\left|\mathrm{C}_{20} \mathrm{H}_{36} \mathrm{~N}_{4} \mathrm{~F}_{2}\right| \\
{ }_{2.49}\left[\mathrm{Si}_{0.90} \mathrm{Ge}_{0.10} \mathrm{O}_{2}\right]_{64}\left[\mathrm{H}_{2} \mathrm{O}\right]_{0.40}\end{array}$ \\
\hline 10BDMI & HPM-7 & 12.22 & 1.90 & 2.62 & ) & $5.44(5.0$ & ) & $10.08(9.0$ & $81.4(82.6)$ & $\begin{array}{c}\left|\mathrm{C}_{20} \mathrm{H}_{36} \mathrm{~N}_{4} \mathrm{~F}_{2}\right| \\
{ }_{2.58}\left[\mathrm{Si}_{0.75} \mathrm{Ge}_{0.25} \mathrm{O}_{2}\right]_{64}\left[\mathrm{H}_{2} \mathrm{O}\right]_{0.42}\end{array}$ \\
\hline
\end{tabular}

aMolar ratios, the theoretical values are given in parentheses. ${ }^{\mathrm{b}}$ Solid residues after thermal analysis to $1000{ }^{\circ} \mathrm{C}$ (the amount in parentheses stands for the $\mathrm{TO}_{2}$ content in a unit cell given in the last column). 'SDA calculated from the $\mathrm{N}$ percent assuming the organics are intact, and are charge-balanced by $\mathrm{F}^{-}$ion, water calculated from the excess $\mathrm{H}$ and $\mathrm{TO}_{2}$ is the rest. ${ }^{\mathrm{d}}$ The sample with the merged two central most intense peaks.

Table 1 lists the chemical composition details of the as-made SDA-zeolites, showing sample variability. For all samples synthesized, there are non-negligible $\mathrm{C} / \mathrm{N}$ ratio deviations from the theoretical values, suggesting partial decomposition might have occurred during the crystallization. There are about 2.5 cationic-like species in one unit cell of the beta-related zeolites calculated based on the $\mathrm{N}$ weight percentage. In order to investigate the existing form of the SDAs occluded in the as-made zeolites, solid state ${ }^{13} \mathrm{C}$ MAS NMR spectra were measured, as shown in Figure 5. The spectra show resonances close to the expected ranges for each $\mathrm{C}$ atom, suggesting the organics are essentially intact in all the samples. However, several resonances overlapped, like the ones centered around $122.6 \mathrm{ppm}$, the $\mathrm{C}$ atoms at the $b$ and $c$ positions on the imidazolium ring, and around $29.7 \mathrm{ppm}$, the $\mathrm{C}$ atoms at $\mathrm{g}, \mathrm{h}, \mathrm{i}$ and $\mathrm{j}$ positions on the methylene chain (see Figure 5 for the C labeling).

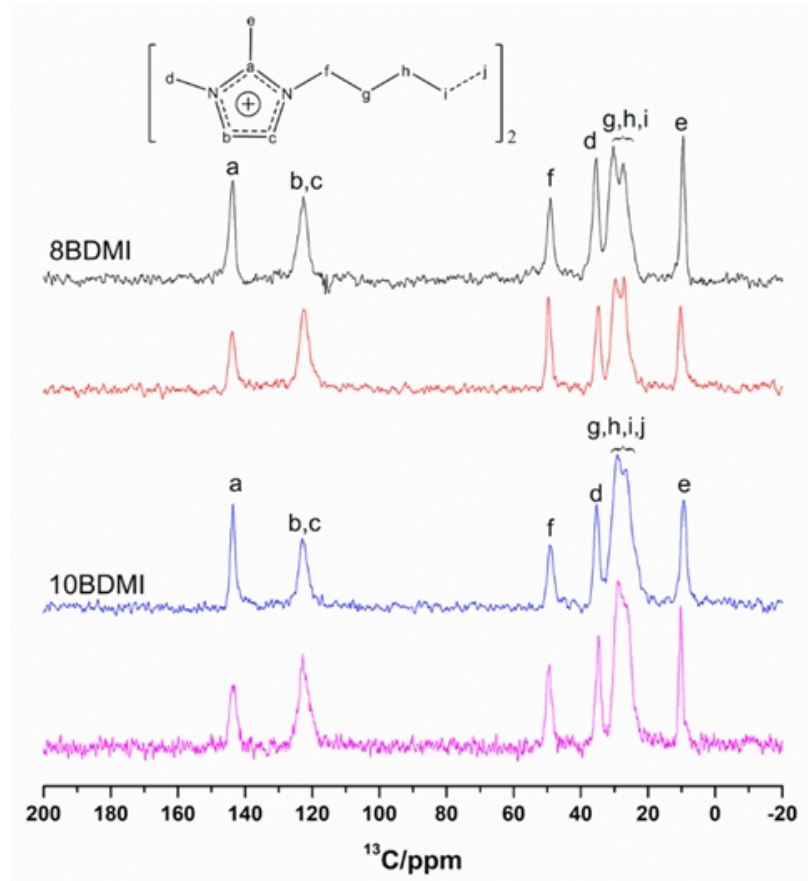

Figure $4 .{ }^{13} \mathrm{C}-{ }^{1} \mathrm{H}$ CPMAS NMR spectra of as-made zeolites using 8BDMI and 10BDMI as SDAs (from bottom to top): 10BDMI-HPM-7-Ge $0.25,10 \mathrm{BDMI}-\mathrm{HPM}-8$ $\mathrm{Ge}_{f} 0.10$, 8BDMI-HPM-7-Ge 0.25 and 8BDMI-HPM-8$\mathrm{Ge}_{f} 0.15$.

Thermogravimetric analysis (TGA) combined with derivative thermogravimetry (DTG) of the samples are shown in Figure S5 and Figure S6. For the samples synthesized with 8BDMI, the amount of organics occluded in the zeolite showed a decreased trend with the increase of $\mathrm{Ge}_{f}$ in the starting gel composition (Table 1 and Figure S5), which has also been observed in our previously reported work. ${ }^{-}$The 10BDMI derived samples also followed the above trend despite the limited $\mathrm{Ge}_{f}$ range tested (Figure S6). This trend is likely due at least in part to the increased fraction of the heavier Ge.

Figure 6 (bottom three traces) shows the ${ }^{19} \mathrm{~F}$ MAS NMR spectra of several as-made HPM-7 zeolites, all showing a single resonance in the -7 to -8 ppm range, which shall be ascribed to $\mathrm{F}$ occluded in D4R units containing Ge pairs with no Ge having more than 2 Ge neighbors. ${ }^{9}$ The spectrum of HPM-7 sharply contrasts with that previously reported for PKU-16, in which $\mathrm{F}=$ not only shows a resonance at $-7.6 \mathrm{ppm}$ assigned to the D4Rs but also presents signals at $-77.2 \mathrm{ppm}$ assigned to $F$ in the lau units and at $-154 \mathrm{ppm}$ assigned to "dissociative $\mathrm{F}^{-\prime}$. These large differences in the ${ }^{19} \mathrm{~F}$ spectra of HPM-7 and PKU-16 may reveal different interactions between the organic cation, the fluoride anion and the zeolite framework. 


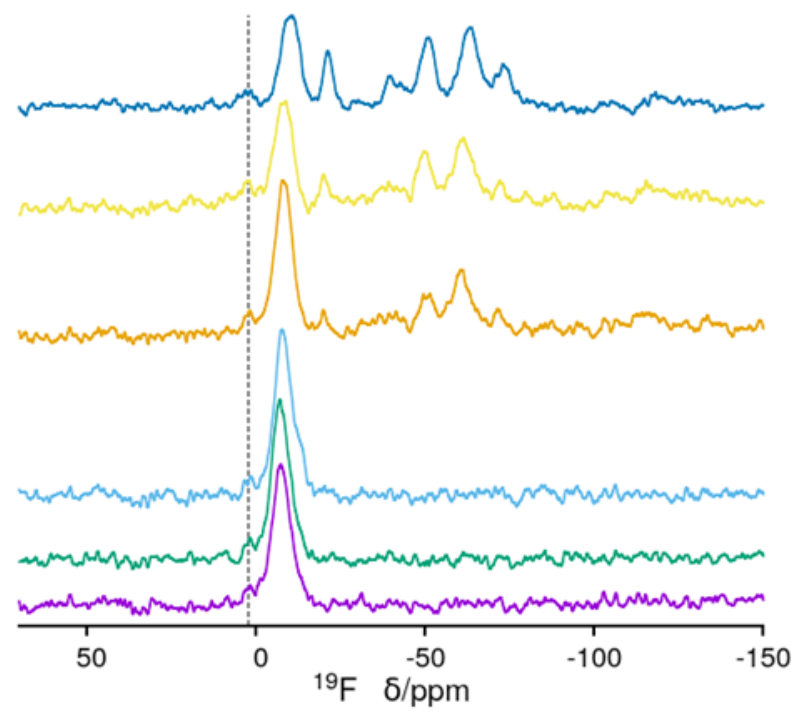

Figure $6 .{ }^{19} \mathrm{~F}$ MAS NMR spectra of (from bottom to top) HPM-7 prepared with 8BDMI $\left(\mathrm{Ge}_{f}=0.20\right.$ and $0.25)$ and 10BDMI $\left(\mathrm{Ge}_{f}=0.25\right)$ and HPM-8 prepared with 8BDMI $\left(\mathrm{Ge}_{f}=0.15\right.$ and 0.10$)$ and 10BDMI $\left(\mathrm{Ge}_{f}=0.10\right)$. The dotted line marks the possible existence of a small resonance at around $2.4 \mathrm{ppm}$, tentatively assigned to $\mathrm{F}$ occluded in open D4R (i.e., D4R with one T-O-T broken bond, see text).

The ${ }^{19} \mathrm{~F}$ spectra of the HPM-8 samples are quite more complicated (Figure 6, top three traces). In addition to a main resonance at similar chemical shift regions, there are three resonances around 50, -61 and -72 ppm, which we attribute to fluoride occluded in other small cavities of the zeolite, such as $\left[4^{3} 5^{4}\right.$ ] (similarly to the case of pure silica zeolite Beta, with resonances at $-58,-65$ and -70 ppm assigned to $F$ in that cavity). ${ }^{24}$ These signals are more prominent for the sample with the lowest Ge fraction. An additional resonance around -20 ppm is assigned to $F$ occluded in D4R containing $\mathrm{Ge}$ but without Ge-O-Ge pairs. 9 This resonance is also more prominent in the sample with lower Ge content, for which still another resonance around -40 pm, assigned to $F$ in pure silica D4R, is also noticeable. For all the samples of HPM-7 and HPM-8 there appears to be a very small resonance at a rather unusual low field $(2.3$ ppm) which we would tentatively assign to $\mathrm{F}$ occluded in defective D4R units, i.e., D4R in which a $\mathrm{T}-\mathrm{O}-\mathrm{T}$ bridge is broken. Resonances at these unusual low fields have been observed in the very recently reported PKU-26 (0.3 ppm), ${ }^{25}$ which contains sti cages (formally a broken D4R), and in STGA-1 (3.9 and 2.2ppm), ${ }^{26}$ where it could be assigned to a similar type of open D4R cage.

\section{Structural Characterization of HPM-8} HRSTEM

Spherical aberration corrected ( $\mathrm{C}_{\mathrm{s}}$-corrected) Scanning Transmission Electron Microscopy (STEM) using both Annular Dark Field and Annular Bright Field detectors were used to characterize the materials obtained. Low-magnification data of

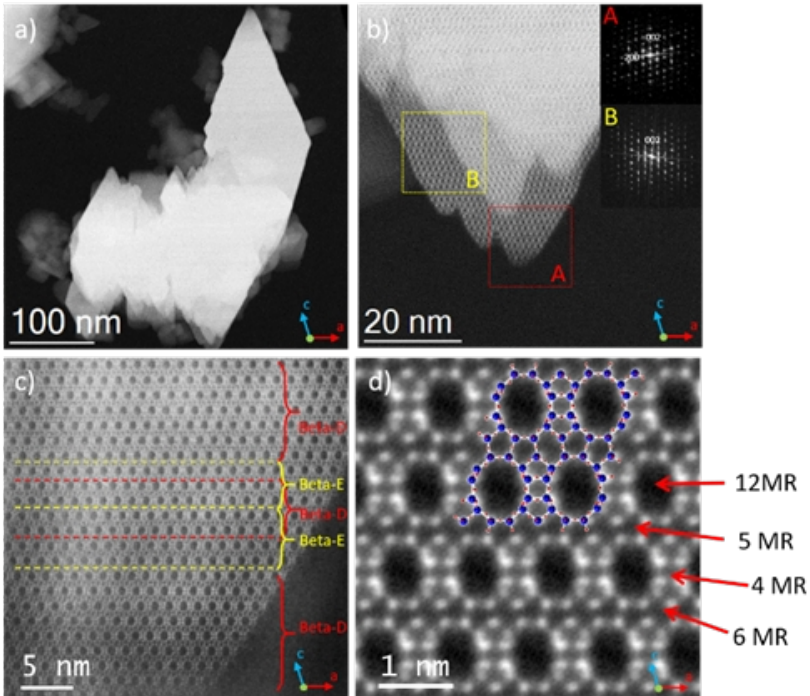

Figure 75. $\mathrm{C}_{\mathrm{s}}$-corrected STEM-ADF analysis of HMP8. a) Low-magnification image displaying the morphology of the crystals. b) Closer observation of the edge of a crystal which allows identifying the defective structure. The FDs shown in the inset confirm the presence of single crystal domains $(A)$ together with faulted regions (B). C) Area of the crystal which permits the identification of the two Beta polymorphs D and E. d) Atomic-resolution data, with the model of the polymorph $D$ overlapped.

HMP-8 reveals well-defined faceted particles with two different morphologies clearly differentiated. Rhombohedral shaped crystals of $100 \mathrm{~nm}$ up to $1 \mu \mathrm{m}$ were identified, see Figure 7a. Such well-defined facets suggest a good crystallinity of the material. Figure 7b displays a closer observation where the pore arrangements showing multiple defects can be clearly appreciated. The Fourier Diffractogram (FD) obtained from two different regions marked as dashed $A$ and $B$ squares are presented. The FD coming from A shows sharp diffraction spots attributed to the single crystal nature of that region; meanwhile, the FD marked as $B$ contains structural defects represented by the diffuse spots that are attributed to the presence of a faulted structure. Figure 7c displays the edge of the crystal in which the two distinct domains can be distinguished and attributed to polymorphs $\mathrm{D}(\mathrm{P} 2 / \mathrm{m})$ and $\mathrm{E}(\mathrm{Pcmm})$ of the Beta family. The crystal orientation can be deduced from these two images as corresponding to the [010], in which the d-spacing measured from the (001) plane distances is $13.3 \AA$ that corresponds to the lattice $c$ constant; the lattice a constant was $\approx$ $11.98 \AA$. Atomic resolution data was also obtained along this orientation, as shown in Figure 7d. In this mode, STEM-ADF, the atomic " $T$ " atoms, Si and $\mathrm{Ge}$, due to their higher atomic number, appear much brighter in comparison to $\mathrm{O}$, which is not observable. Therefore, from Figure $7 d$, it is possible to infer the structure of this material, locating the $n$-membered rings ( $n \mathrm{MR}$ ) that conform the structure (12MR, 6MR, 5MR and 4MR). The structural model for polymorph $D$ of the Beta family has been superimposed where " $T$ " and $O$ 


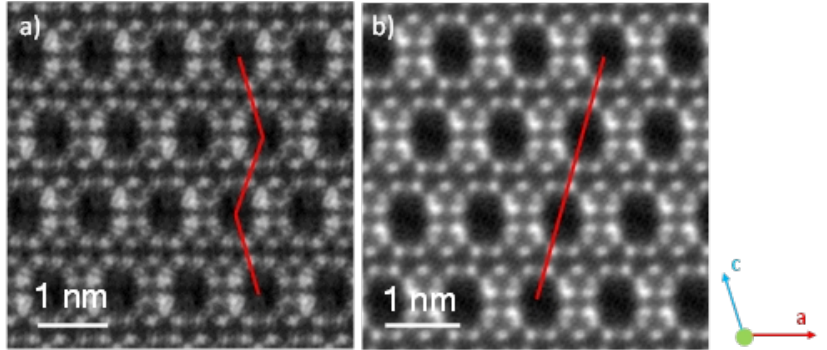

atoms are represented as blue and red spheres, respectively, corroborating the perfect matching between the model of polymorph $\mathrm{D}$ and the experimental data acquired. The two polymorphs can be described as two stacking sequences, in which the 12 MR of the polymorph E follows a zig-

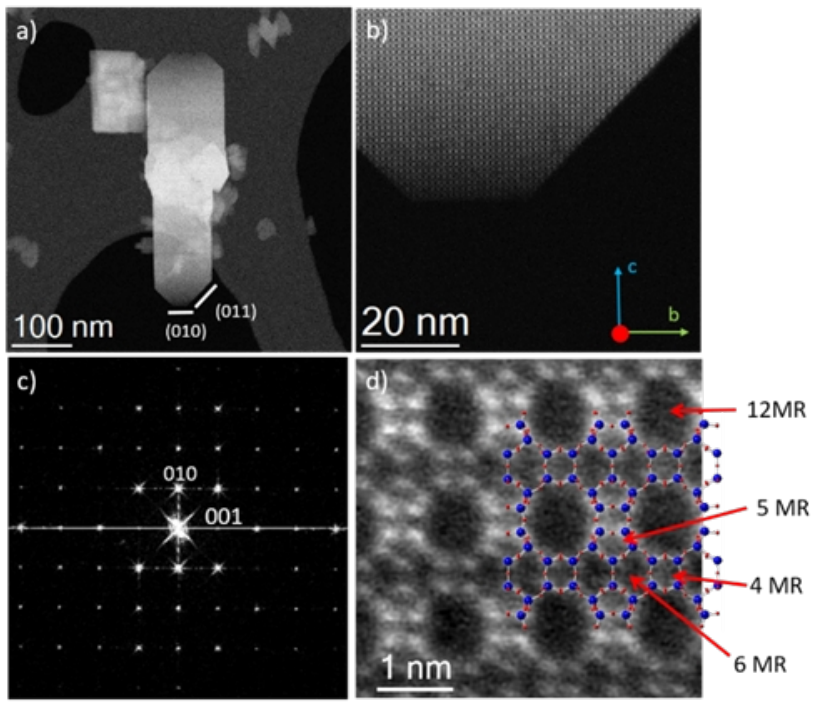

zag trend along the $c$ axis (see Figure 8a), where every other layer is shifted approximately $1 / 3$ of a, $4 \AA$, (i.e., shifts are $0,1 / 3,0,1 / 3 \ldots$ ) whereas for polymorph $D$ successive layers are always shifted by $1 / 3 a$ (i.e., shifts are $0,1 / 3,2 / 3,0,1 / 3 \ldots$ ), as seen in Figure $8 b$.
Despite the numerous defects that arise as a consequence of the coexistence of both polymorphs, when the crystals are observed perpendicular to this projection (this is along [100]) the materials exhibit a single crystal nature where the defects are not observable. This is due to the fact that both polymorphs $D$ and $E$ share a common projection along [100] (which is also common to polymorph $\mathrm{C}$ ). In this case, the low-magnification micrograph reveals the morphology along this projection of HMP-8, elongated crystals with rectangular shapes which generally terminate with truncated ends corresponding to the (011) and (010) facets, see Figure 9a. High-resolution studies by means of $\mathrm{C}_{\mathrm{s}}$-corrected STEM-ADF were also performed along this zone axis. Figure $9 \mathrm{~b}$ corresponds to the crystal edge that allows the observation of the pore distribution as well as extracting the FD diffractogram, Figure 9c. The FD can be indexed assuming $P c m m$ or $P 2 / m$ obtaining a lattice constant $b=12.9 \AA$ and $c=13.47 \AA$. The atomic resolution data is depicted in Figure 9d, where all T atoms can be identified corroborating that along this projection the framework is identical as BEC.

Thus, we can observe in HPM-8 defect-free projections that are shared by polymorphs C, D and $E$ as well as faulted projections in which polymorphs $A$ or $E$ are intergrown with polymorphs $B$ or $D$ respectively, as expected for an intergrowth between polymorphs $\mathrm{D}$ and $\mathrm{E}$. To unambiguously determine the HPM-8 structure three dimensional diffraction electron tomography (3D-EDT) was collected from several crystals obtaining in every case the same result. The typical data acquired for this material is presented in figure S7. Figure S7a and S7b depict two different orientations related by $90^{\circ}$ of the same crystal which correspond to the [100] and the [010] projections. The shape of the crystals of these tow images are in agreement with the images presented in figures 7 and 8 that correspond to HPM-8. The electron diffraction analysis by means of 3D-EDT, figures S7c and S7d shows two electron diffraction patterns extracted from the reconstructed three dimensional diffraction data along the $a *$ and $b *$ orientations. From these two ED patterns it is clear the absence of structural defects when the HPM-8 is along the [100] orientation, that, however appear when the crystal was tilted evidenced by the existence of the diffuse spots, as streaks, on the [010] orientation. Lattice cell parameters obtained by this method were $\mathrm{a}=12.72 \AA ; \mathrm{b}=$ $12.89 \AA ; c=14.22 \AA ; \alpha=89.89^{\circ} ; \beta=107.02^{\circ} ; \gamma=$ $90.14^{\circ}$ for a space group $P 2 / m$ in good agreement with the predominant polymorph $D$.

Thus, we conclude that HMP-8 is a highly crystalline material formed by an intergrowth of polymorphs $D$ and $E$ where, after observations of over 15 different crystallites, polymorph $\mathrm{D}$ is the predominant phase as evidenced in Figure 7. 
In order to further characterize the structure of HPM-8, we simulated the powder XRD data of intergrowths between polymorphs $D$ and $E$ of the Beta family (the recently reported SU-78B and SU-78A, respectively) ${ }^{14}$ using DIFFaX. ${ }^{27}$ The layers were obtained from the reported SU-78A structure, ${ }^{14}$ but centering the so-called "helicopter" in the center of the cell. The length of the cell edges was deduced from the experimental pattern of calcined HPM-8 (reflections that are invariant when polymorphs $D$ and $E$ intergrow were used to refine the cell). We checked that only 2 layers (related by a $90^{\circ}$ rotation) are necessary to build all the Beta family of polymorphs (A to E). Both layers need to strictly alternate, while they may be or not shifted by $\pm 1 / 3$ of the corresponding cell edge along $x$ or $y$, depending on the polymorph. Along $z$ a shift of $1 / 4$ of the $c$ edge needs to be applied to each successive layer. Figure 10 shows the effect of intergrowing polymorphs $D$ and $E$ on the XRD pattern and compares the patterns with the simulation for the pure polymorphs and the experimental pattern of HPM-8.

The most visible change in the pattern along this intergrowth refers to reflection (101) of the orthorhombic polymorph E (SU-78A), at around $7.8^{\circ}$, which splits into two broader reflections that are more separated as the prevalence of polymorph D (SU-78B) increases, finally becoming reflections (100) and (10-1) of this monoclinic polymorph (at around 7.4 and $8.3^{\circ}$ in the pure polymorph). Comparison with the experimental pattern suggests that, contrarily to SU-78, HPM-8 is significantly enriched (around $80 \%$ ) in polymorph D. Further comparison in the $76-86 \%$ percent range suggests HPM- 8 is an intergrowth of polymorphs $\mathrm{D}$ and $\mathrm{E}$ in around an 82:18 ratio (Figure S8).

When we compare HPM-8 samples displaying less well resolved patterns with the simulations, the positions and general features of the peaks can be reproduced well with an intergrowth containing $84 \% \mathrm{D}$, see Figure S9. The cell in this case was a little bit smaller $(a=12.5645 \AA$, $b=12.6787 \AA, c=26.51984 \AA$ ). Thus, the presence of a single central reflection rather than two sharper lines in HPM-8 turned out to be simply a consequence of the change in cell parameters and increase in peak broadness, which made the two reflections to merge into a broad and asymmetric one.

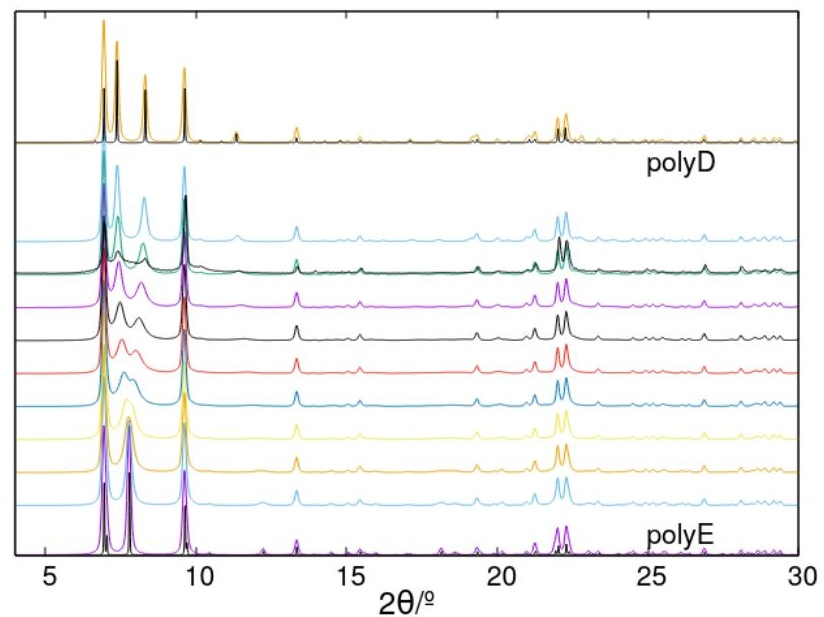

Figure 8. DIFFaX simulated powder XRD patterns of intergrowths of polymorphs $E$ and $D$ of the Beta family (color traces, with $10 \%$ increments). The bottom and top traces correspond to $99 \%$ pure polymorphs $E$ and $D$, respectively, and are compared to a simulation of the corresponding polymorph obtained with the program VESTA. ${ }^{28}$ The experimental pattern for a HPM-8 sample is shown (noisy black trace) close to the $80 \%$ polymorph $\mathrm{D}$ simulation. The layer used in

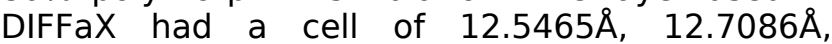

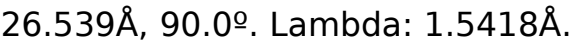

We note that all the reflections in the experimental pattern of HPM-8 are somehow broader than those in the simulations. Tweaking with peak broadening parameters in DIFFaX (U, V, W and Gaussian-Lorentzian mixing parameter) did not solve this difference: while all experimental peaks are broad, this is by far more apparent in the peaks that are affected by the intergrowth (while the $\mathrm{U}, \mathrm{V}, \mathrm{W}$ and mixing parameters affect all the peaks). Playing with the number of stacked layers or with the widths of the layers did not help either because the effect on the pattern was relatively small. This suggested that additional disorder may occur in HPM-8. Given that $\mathrm{C}_{\mathrm{s}}$-corrected STEM data shows the coexistence of projections that are compatible with polymorphs A, B and C (in addition to $D$ and $E$ ), we introduced variable proportions of these polymorphs into the DE intergrowth simulation, which did not result in an improvement in the match (see Figures S10-S13). However, since an exhaustive simulation of the intergrowth of 3 or 4 polymorphs covering all range of possible relative amounts would be extremely time consuming, we cannot discard this possibility and, in fact, we have observed by HRSTEM a single occasion in which projections compatible with $C$ (or $D$ or $E$ ) intergrew with projections compatible with $A$ and $B$ (or $D$ and $E$ ), see Figure S14. While this fact by itself does not prove or disprove the presence of additional polymorphs in HPM-8, we think there may be some additional disorder in this system that we can not specify. Anyway, we can safely conclude that HPM-8 is basically an intergrowth of polymorphs $D$ and $E$ much enriched in polymorph $D$ (around $80 \%)$ compared to SU-78 (50\%). Another difference is that HPM-8 is significantly richer in $\mathrm{Si}$, as deduced not only from the lower $\mathrm{Ge}_{f}$ in the gel (0.1-0.2 compared to 0.4 in SU-78) but also from the smaller cell ( $4231-4225 \AA^{3}$ compared to the reported $4313 \AA^{3}$ for SU-78).

\section{Computational results}

In order to investigate the structure-directing effect of these long dications, we finally analyzed by molecular mechanics simulations their fit with the zeolite void volume. The POS framework (corresponding to HPM-7) contains three channel systems, one 12MR channel running parallel to $\mathrm{c}$, and two 11MR channels running perpendicular to $c$, and perpendicular to each other, with an elliptical shape and a slightly smaller cross-section. We 
first introduced manually the 8BDMI dications in the two types of POS channel systems, the $12 \mathrm{MR}$ and 11MR channels (the two 11MR are topologically equivalent), and calculated the most stable location by simulated annealing; as we did before with STW, $\underline{5}$ the OSDA dications were loaded in the two possible orientations of the imidazolium rings, with methyl groups of the two rings pointing to the same side ('ss') or to opposite sides ('ops'). In order to have enough space to accommodate the long 8BDMI species along the $12 \mathrm{MR}$ channel, $1 \times 1 \times 2$ supercell systems were used (' $a$ ' and ' $b$ ' directions are large enough as to host one dication while preventing interactions between image cells). Two possible locations for 8BDMI are possible within the 11MR channels, with the imidazolium rings in the intersections with the $12 \mathrm{MR}$ (labelled as 'intersections'), or with the alkyl spacer sited in those intersections, and the imidazolium rings close to the D4Rs (labelled as 'D4R'). The relative energies are given in Table S3, and images of some of the models (those with 'ss' orientation) are shown in Figure S16.

From the energy results, it is clear that the 8BDMI dication fits better within the 11MR channels, probably because of a stronger confinement and hence higher interaction with the channel walls due to their smaller cross-section; worth is noting that this type of dications typically structure-direct towards 10MR channels, like those in MFI or STW. On the other hand, the location of the dications with the imidazolium rings sited close to the D4Rs is rather more stable (Figure S16-bottom), again due to a stronger confinement with both D4R units surrounding the imidazolium rings than in the more open channel intersections (Figure S16-middle).

In the next model, two 8BDMI dications were included in the most stable location (11MR channels). As there are two of these channels per POS u.c. (perpendicular to each other), we loaded one dication on each 11MR channel, giving a packing of 2 SDAs per u.c. 8BDMI cations were loaded in the most stable site, with the imidazolium rings close to the D4Rs, and in the two possible conformations ('ss' and 'ops'). In addition, there are two possible arrangements with the 2 dications in the 11MR channels: in the first (referred as ' $A$ ', Figure S17-left), one intersection with the 12MR channel is filled with the spacers of the 2 dications, while leaving free the other 12MR channel, while in the other (referred as ' $B$ ', Figure S17-right), the 8BDMI dications are shifted and hence the two $12 M R$ channels are only partially filled with the spacers; the resulting relative energies are given in Table S4. The most stable arrangement corresponds to the ' $A$ ' configuration, where one of the 12MR channels is occupied by the spacers of the two dications, and the other remains empty (see Figure S17-left); the most stable orientation of the imidazolium rings in this case is in the 'ops' orientation (see Table S4), giving a rather symmetric arrangement of the dications which nicely fits with the POS topology. It should be remarked that this configuration enables the incorporation of further dications within the empty $12 \mathrm{MR}$ channel (see void volume surface in Figure S17-left). In the ' $B$ ' configuration, which is less stable (Table S4), each $12 \mathrm{MR}$ channel is partially filled with the organic spacers, and hence does not allow for additional dications loading (see Figure S17-right).

As previously mentioned, the ' $A$ ' organic arrangement allows for the inclusion of one additional 8BDMI dication along the empty 12MR channel. In this case, $1 \times 1 \times 2$ supercell systems are required in order to accommodate the 8BDMI dication aligned with ' $c$ ' direction (12MR channel); hence, 5 dications were loaded in the $1 \times 1 \times 2$ supercell as follows: 4 ( 2 per unit cell) in the $11 \mathrm{MR}$ channels in the A-ops configuration, and one additional dication aligned with the empty 12MR channel, both in 'ss' and 'ops' orientations. Again the most stable situation was found for the system where the 8BDMI dication in the 12MR channel was in the 'ops' orientation. Figure S18 shows the final arrangement of the 8BDMI dications, giving a packing value of $5 / 2=2.58$ BDMI/u.c., in close agreement with the value found experimentally.

Calculations with the longer 10BDMI dication within the POS framework (in the ' $A$ ' configuration) gave a similar location for this SDA (Figure $\mathrm{S} 19)$. We note that in both cases (8BDMI and 10BDMI dications, Figures S17 and S19, respectively), the imidazolium rings always site very close to a D4R unit, which may be possible for both dications due to the inherent flexibility of the spacer.

We then studied the location of the 8BDMI dications within a hypothetical pure polymorph $D$ of zeolite Beta (as a representation of HPM-8). In this case, one 8BDMI dication was loaded in a $2 \times 2 \times 2$ supercell in three different orientations, with the molecular axis aligned with the [100], [010] or [001] channels, and again in 'ss' or 'ops' conformations of the imidazolium rings. Table S5 reports the final relative energies, and Figure S20 displays the final configuration for the 'ops' conformations. The most stable location for 8BDMI is when they site aligned with the [001] channels (Figure S20-bottom); the next more stable orientation is with 8BDMI aligned with the [010] channels, while the least stable is aligned with the [100] channels. We note that in the two most stable orientations (along [010] and [001], Table S5), the 8BDMI dications site with the two imidazolium rings very close to D4R units (Figure S20-middle and bottom), the same as occurred in the POS framework. 
Figure 9. Relationship between the RDF between all atoms of the imidazolium rings of 8BDMI (blue lines) and 10BDMI (red lines) and the inter-D4R distance in POS (vertical black dashed lines) and polymorph $\mathrm{D}$ of beta (vertical grey dashed lines).It is interesting to remark the tendency of these dications to locate their imidazolium rings close to D4R units in both frameworks (POS and beta polymorph $\mathrm{D}$ ). We note here that this conclusion arises from a calculation without occluded fluoride so it must be topological in nature, and that it would be reinforced when considering electrostatic interactions between fluoride-containing D4R and the charged imidazolium rings. From a chemical point of view, fluoride and imidazolium rings are expected to be closely associated during the crystallization process. In fact, it seems that the efficient structure-directing effect of these long dications towards these particular frameworks is closely associated to the adequate distance match between adjacent D4R units in both frameworks and the two imidazolium rings in these long dications. Adjacent D4Rs in the POS framework are separated by $13.77 \AA$ along the $11 M R$ channels (the most stable location for 8BDMI, Table S4) and by $11.69 \AA$ along the $12 \mathrm{MR}$ channels (the less stable position for 8BDMI), while those inter-D4R distances in polymorph $D$ of

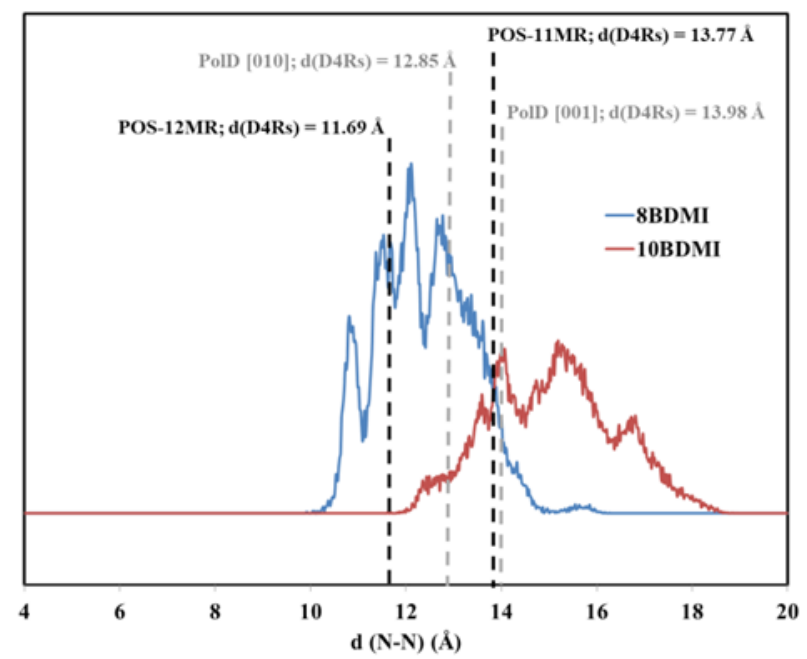

zeolite Beta are $12.85 \AA$ along the [010] channels and $13.98 \AA$ along the [001] channels, the two most stable locations for 8BDMI (Table S5). In order to monitor such geometric match, we compared these inter-D4R distances with the intramolecular distance between the two imidazolium rings in the two dications. In order to have averaged values, we ran 500 ps of Molecular Dynamics of the dications in vacuum, and plot the Radial Distribution Functions of these inter-ring distances (Figure 11). We can observe that the range of distances between imidazolium rings falls close to the distance between adjacent D4R units in both frameworks, especially in the 11MR channel direction in POS (13.77 $\AA$ ) and in the [001] channels in polymorph D (13.98 Å), for both cations. Indeed, this geometric relationship is even more appealing when considering the dis- tance between $\mathrm{N}$ atoms (Figure S21), which should be an indication of the separation between the positive charges; two limits are defined, the lowest one is given by the distance between the internal $\mathrm{N}$ atoms ( $\mathrm{N}_{\text {int }}, \mathrm{N}$ bonded to the alkyl spacer), and the highest one is given by the maximum separation of charges as represented by the distance between external $\mathrm{N}$ atoms $\left(\mathrm{N}_{\text {ext }}, \mathrm{N}\right.$ bonded to methyl groups). Again we observe a perfect distance match between external $\mathrm{N}$ atoms of 8BDMI (solid blue lines in Figure S28) and the inter-D4R separation in the POS framework along the 11MR channels (and this can explain why these SDAs prefer to site along the 11MR channels), and also with the inter-D4R separation in beta polymorph D along the [001] channels (and also is not far from the separation along [010]). In the case of 10BDMI, the distance between external $\mathrm{N}$ atoms (solid red line) is longer $(\sim 16.7 \AA)$ than the inter-D4R separation (13.8-14 $\AA$ ), but in contrast the distance between the internal $\mathrm{N}$ atoms (dashed red line) falls right in the interD4R separation of both POS and beta polymorph D. In any case, we note that the positive charge of imidazolium rings in principle should be delocalized around the ring, but is possible that the charge might localize on a particular $\mathrm{N}$ atom upon interaction with the D4R units and the occluded fluoride.

\section{CONCLUSION}

Introduction of small amounts of $\mathrm{Ge}$ in a synthesis using dimethylimidazolium-based long dications that would otherwise yield the well-known and trite MTW produces interesting changes in the phase selectivity of the crystallization. As the $\mathrm{Ge}_{f}$ increases, *BEA, HPM-8 and HPM-7 are obtained. The trend corresponds to an increase in the density of D4R. *BEA has no D4R (although it is difficult to discard the occurrence in this intergrown material of small proportions of polymorphs $C, D$ and $\mathrm{E}$, all containing D4R), while HPM-8 and HPM7 contain 2 and 4 D4R, respectively, per $64 \mathrm{~T}$ atoms.

HPM-8 is an intergrowth of polymorphs $D$ and $E$ of the zeolite Beta family with a large predominance (over $80 \%$ ) of polymorph $D$, as deduced from DIFFaX simulations and HRTEM. Compared to the recently reported SU-78, which contains an almost $1: 1$ proportion of both $D$ and $E$ polymorphs, HPM-8 is significantly more stable due to its much lower $\mathrm{Ge}$ content $\left(\mathrm{Ge}_{f}=0.1-0.15 \mathrm{com}\right.$ pared to 0.3 ). On the other hand, HPM-7 is likely isostructural to POS in view of its PXRD data.

Computer simulations confirm that 8BDMI can accommodate in the POS channels in a high loading of up to 2.5 dications per unit cell and show that the most favorable host-guest interaction is within the 11MR channels (2 dications per cell), with the imidazolium moieties in between D4R units providing a strong confinement, and with an additional loading in the 12MR (up to 0.5 dications per cell) being possible. Interestingly, also in 
the framework of polymorph $D$ of zeolite beta do 8BDMI dications site with imidazolium rings close to D4R units. In fact, our results suggest that the efficiency of these long organic dications to direct the crystallization of these frameworks is closely associated to the appropriate distance match between the positive charges of the imidazolium rings in 8BDMI and 10BDMI dications, determined by the length of the alkyl spacers, and adjacent D4R units in these germanosilicate zeolites.

\section{ASSOCIATED CONTENT}

Supporting Information. Tabulated results for the synthesis of germanosilicate zeolites, textural properties of the calcined zeolites, ${ }^{1} \mathrm{H}$ and ${ }^{13} \mathrm{C}$ NMR in $\mathrm{D}_{2} \mathrm{O}$ of the two imidazolium dications, extra XRD patterns for HPM-7, TG-DTG curves for HPM-7 and HPM-8 samples, the comparison of experimental XRD pattern of HPM-8 with the DIFFaX simulated patterns of intergrowths of polymorphs $D$ and $E$ in the $76-86 \%$ polymorph $\mathrm{D}$ range, the comparison of the experimental pattern of HPM-8 with the DIFFaX simulated patterns of intergrowths of polymorphs $D$ and $E$ in a 4:1 ratio with variable proportions of polymorph $A / B /$ $A \& B$, Rietveld refinement plot and results, SEM and TEM images and analyses of HPM-8 and HPM-7 samples, IR spectroscopy data of as-made samples in comparison with traditional beta zeolite, locations of 8BDMI and 10BDMI in the channels of HPM-8 and POS as determined by molecular mechanics, illustration of the relationship between the $\mathrm{N}-\mathrm{N}$ distances of the dications and the inter-D4R distances. This material is available free of charge via the Internet at http://pubs.acs.org.

\section{AUTHOR INFORMATION}

\section{Corresponding Author}

* E-mail: macamblor@icmm.csic.es

\section{Present Addresses}

†Johns Hopkins University, 3400 Charles ST, Institute for Nanobiotechnology/WSE, Baltimore, MD, USA.

\section{Notes}

The authors declare no competing financial interest.

\section{ACKNOWLEDGMENT}

The authors acknowledge financial support by the Spanish Ministry of Science, Innovation and Universities (projects MAT2016-77496-R and MAT201571117-R) and the National Natural Science Foundation of China (NSFC-21850410448, NSFC21835002). P.L. is grateful to the China Scholarship Council (CSC) for a fellowship and to the Dalian Institute of Chemical Physics for permission to leave. AM acknowledges to The Centre for High-resolution Electron Microscopy (CћEM), supported by SPST of ShanghaiTech University under contract No. EM02161943. We are also indebted to the ERSF (Grenoble) and the BM25 Spline staff, particularly to G. Castro and A. Serrano and to L. A. Villaescusa (Valencia) for help in collecting the synchrotron XRD data and for helpful comments and suggestions. Centro Técnico Informático-CSIC is acknowledged for running the calculations, and Biovia for providing the computational software.

\section{REFERENCES}

(1) Davis, M. E.; Lobo, R. F., Zeolite and Molecular Sieve Synthesis. Chem. Mater. 1992, 4, 756-768.

(2) Camblor, M. A.; Villaescusa, L. A.; Díaz-Cabañas, M. J., Synthesis of All-silica and High-silica Molecular Sieves in Fluoride Media. Top. Catal. 1999, 9, 59-76.

(3) Flanigen, E. M.; Patton, R. L. US4,073,865, 1978.

(4) Lu, P.; Villaescusa, L. A.; Camblor, M. A., Driving the Crystallization of Zeolites. Chem. Rec. 2018, 18, 713-723.

(5) Lu, P.; Gómez-Hortigüela, L.; Xu, L.; Camblor, M. A., Synthesis of STW Zeolites Using Imidazolium-based Dications of Varying Length. J. Mater. Chem. A 2018, 6, 1485-1495.

(6) Lu, P.; Gómez-Hortigüela, L.; Camblor, M. A., Synthesis of Pure Silica MWW Zeolite in Fluoride Medium by Using an Imidazolium-Based Long Dication. Chem. Eur. J. 2019, 25, 1561-1572.

(7) Zones, S. I.; Darton, R. J.; Morris, R.; Hwang, S.-J., Studies on the Role of Fluoride Ion vs Reaction Concentration in Zeolite Synthesis. J. Phys. Chem. B 2005, 109, 652-661.

(8) Wang, Y.; Song, J.; Gies, H., The Substitution of Germanium for Silicon in AST-type Zeolite. Solid State Sci. 2003, 5, 1421-1433.

(9) Rigo, R. T.; Balestra, S. R. G.; Hamad, S.; BuenoPerez, R.; Ruiz-Salvador, A. R.; Calero, S.; Camblor, M. A., The Si-Ge Substitutional Series in the Chiral STW Zeolite Structure Type. J. Mater. Chem. A 2018, 6, 1511015122.

(10) Rojas, A.; Martínez-Morales, E.; Zicovich-Wilson, C. M.; Camblor, M. A., Zeolite Synthesis in Fluoride Media: Structure Direction toward ITW by Small Methylimidazolium Cations. J. Am. Chem. Soc. 2012, 134, 22552263. R2.

(11) Forcite Module. BIOVIA Materials Studio, 2017

(12) Dauber-Osguthorpe, P.; Roberts, V. A.; Osguthorpe, D. J.; Wolff, J.; Genest, M.; Hagler, A. T., Structure and Energetics of Ligand Binding to Proteins: Escherichia Coli Dihydrofolate Reductase-trimethoprim, A Drug-receptor System. Proteins Struct. Func. Genet. 1988, 4, 31-47.

(13) http://iza-structure.org/databases/. Accessed on 02/01/2019.

(14) Yu, Z.-B.; Han, Y.; Zhao, L.; Huang, S.; Zheng, Q.Y.; Lin, S.; Córdova, A.; Zou, X.; Sun, J., Intergrown New Zeolite Beta Polymorphs with Interconnected 12-Ring Channels Solved by Combining Electron Crystallography and Single-Crystal X-ray Diffraction. Chem. Mater. 2012, 24, 3701-3706.

(15) Gemmi, M., Oleynikov, P., Scanning Reciprocal Space for Solving Unknown Structures: Energy Filtered Diffraction Tomography and Rotation Diffraction Tomography Methods. Z. Kristallogr. Cryst. Mater. 2013, 228, 51-58.

(16) Newsam, J. M.; Treacy, M. M. J.; Koetsier, W. T.; De Gruyter, C. B.; Thomas, J. M., Structure Characterization of Zeolite Beta. Proc. R. Soc. London. Ser. A 1988, $420,375-405$

(17) Burton, A. W.; Elomari, S.; Chan, I.; Pradhan, A.; Kibby, C., Structure and Synthesis of SSZ-63: Toward an Ordered Form of Zeolite Beta. J. Phys. Chem. B 2005, 109, 20266-20275.

(18) Hua, W.; Chen, H.; Yu, Z.-B.; Zou, X.; Lin, J.; Sun, J., A Germanosilicate Structure with $11 \times 11 \times 12$-Ring Channels Solved by Electron Crystallography. Angew. Chem. Int. Ed. 2014, 53, 5868-5871.

(19) Valencia, S. Nuevos Métodos de Síntesis de Zeolita Beta: Obtención del Isomorfo SiO2 y Sustitución 
de Si por Al, Ti y Ge. Universitat Politècnica de València, 1997.

(20) Blasco, T.; Corma, A.; Díaz-Cabañas, M. J.; Rey, F.; Vidal-Moya, J. A.; Zicovich-Wilson, C. M., Preferential Location of $\mathrm{Ge}$ in the Double Four-Membered Ring Units of ITQ-7 Zeolite. J. Phys. Chem. B 2002, 106, 26342642.

(21) Corma, A.; Díaz-Cabañas, M. J.; Jiang, J.; Afeworki, M.; Dorset, D. L.; Soled, S. L.; Strohmaier, K. G., Extra-large Pore Zeolite (ITQ-40) with the Lowest Framework Density Containing Double Four- and Double Three-rings. Proc. Natl. Acad. Sci. U.S.A. 2010, 107, 13997-14002.

(22) Bai, R.; Sun, Q.; Wang, N.; Zou, Y.; Guo, G.; Iborra, S.; Corma, A.; Yu, J., Simple Quaternary Ammonium Cations-Templated Syntheses of Extra-Large Pore Germanosilicate Zeolites. Chem. Mater. 2016, 28, 6455-6458.

(23) Boultif, A.; Louer, D., Powder Pattern Indexing with the Dichotomy Method. J. Appl. Crystallogr. 2004, 37, 724-731.

(24) Camblor, M. A.; Barrett, P. A.; Díaz-Cabañas, M. a.-J.; Villaescusa, L. A.; Puche, M.; Boix, T.; Pérez, E.; Koller, H., High silica zeolites with three-dimensional systems of large pore channels. Microporous Mesoporous Mater. 2001, 48, (1), 11-22.

(25) Chen, Y.; Ma, N.; Huang, S.; Du, X.; Liao, F.; Zhu, Y.; Sun, J.; Wang, Y., An Interrupted Zeolite PKU-26 and Its Transformation to a Fully Four-Connected Zeolite PKU-27 upon Calcination. Chem. Eur. J. 2019, 25, 32193223.

(26) Villaescusa, L. A.; Wheatley, P. S.; Morris, R. E.; Lightfoot, P., A Severely Interrupted Germanate Zeolite Framework Synthesised from Isolated Double Four-ring Units. Dalton Trans. 2004, 0, 820-824.

(27) Treacy, M. M. J.; Newsam, J. M.; Deem, M. W., A General Recursion Method for Calculating Diffracted Intensities from Crystals Containing Planar Faults. Proc. R. Soc. London. Ser. A 1991, 433, 499-520.

(28) Momma, K.; Izumi, F., VESTA 3 for Three-dimensional Visualization of Crystal, Volumetric and Morphology Data. J. Appl. Crystallogr. 2011, 44, 1272-1276. 